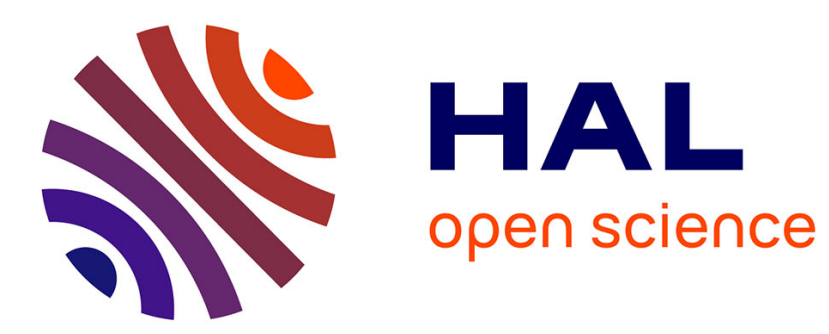

\title{
Quelques éléments de réflexion sur l'incertitude à travers l'histoire des sciences et des idées.
}

\author{
Alexandre Moatti
}

\section{To cite this version:}

Alexandre Moatti. Quelques éléments de réflexion sur l'incertitude à travers l'histoire des sciences et des idées.. Annales des mines - Série Responsabilité et environnement, 2010, 57, p. 16-21. halshs00449621

\section{HAL Id: halshs-00449621 \\ https://shs.hal.science/halshs-00449621}

Submitted on 22 Jan 2010

HAL is a multi-disciplinary open access archive for the deposit and dissemination of scientific research documents, whether they are published or not. The documents may come from teaching and research institutions in France or abroad, or from public or private research centers.
L'archive ouverte pluridisciplinaire HAL, est destinée au dépôt et à la diffusion de documents scientifiques de niveau recherche, publiés ou non, émanant des établissements d'enseignement et de recherche français ou étrangers, des laboratoires publics ou privés. 


\section{RESPONSABILITÉ \& ENVIRONNEMENT recherches débats actions}

Faire face à l'incertitude

JANVIER 2010

NUMÉRO 57

PRIX : $23 €$

ISSN $1268-4783$

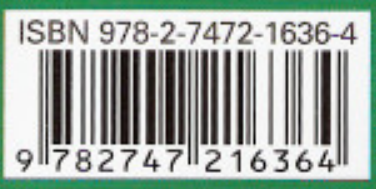

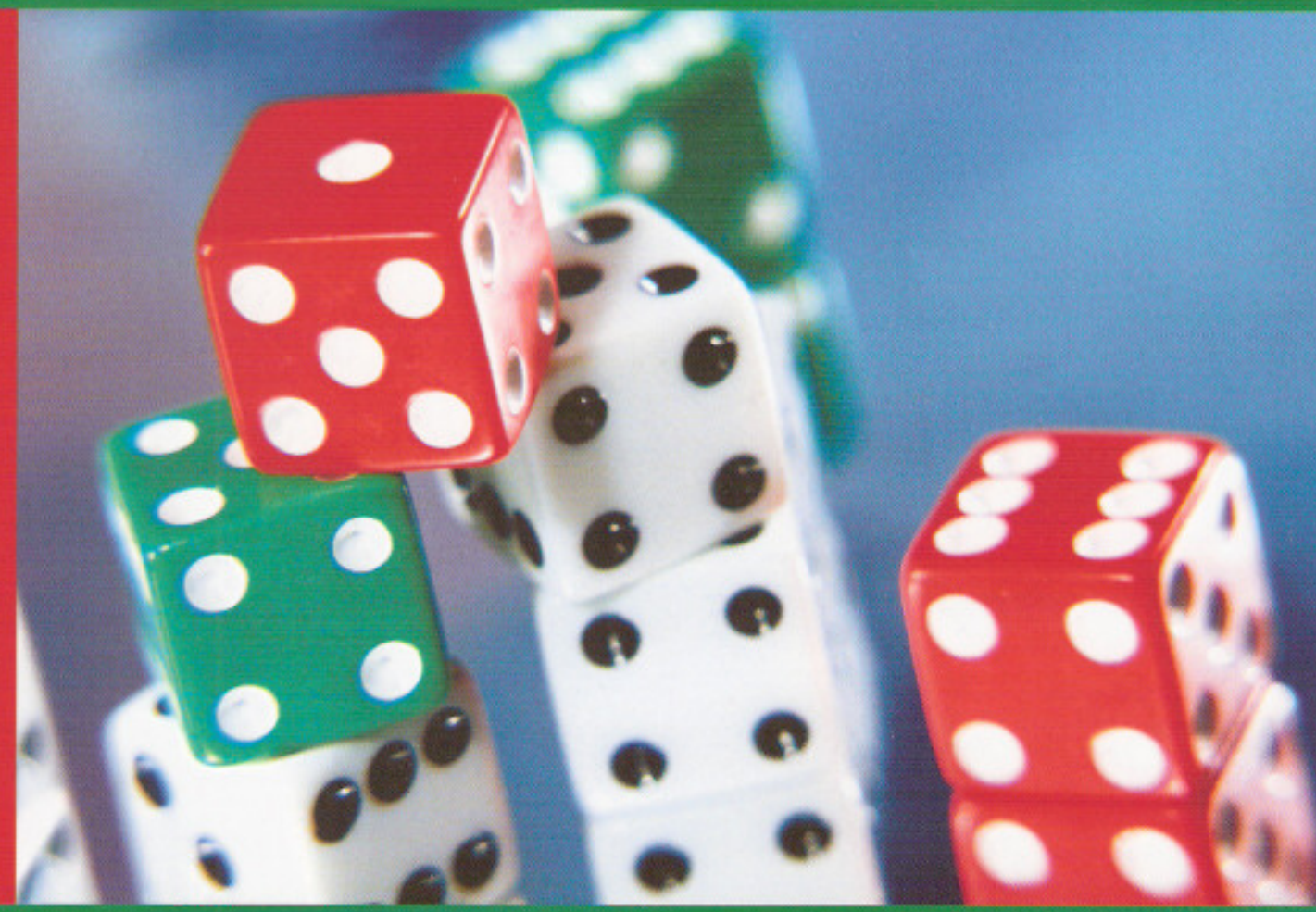

SÉRIE TRIMESTRIELLE DES

ANNALES

DES

MINES

FONDÉES EN 1794 


\section{SOMMAIRE}

\section{FAIRE FACE À L'INCERTITUDE}

5 ÉDITORIAL

Pierre Couneinhes

7 Avant-Propos

L'homme en quête de certitudes : entre croyance et savoir

Marie-Josèphe CARRIEU-COSTA

\section{I - Quelques aspects théoriques}

\section{sur l'incertitude}

\section{0}

Penser les événements extrêmes Jean-Pierre Dupur

\section{6}

Quelques éléments de réflexion sur l'incertitude à travers l'histoire des sciences et des idées Alexandre MoATI

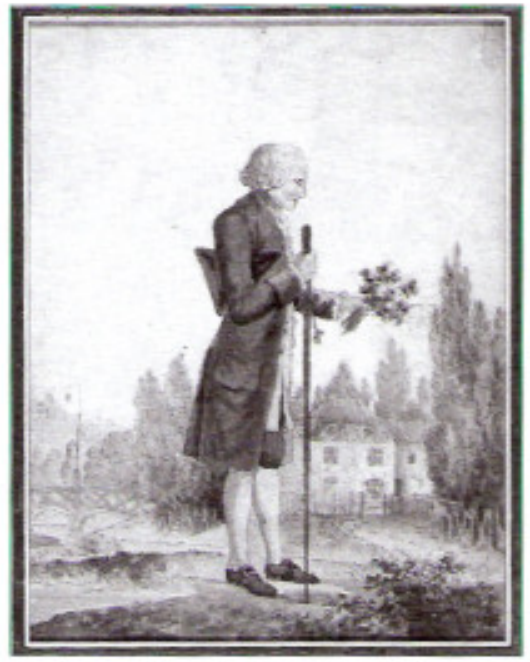

- Paris, Musée Camavalet//ROGER-VIOLLE

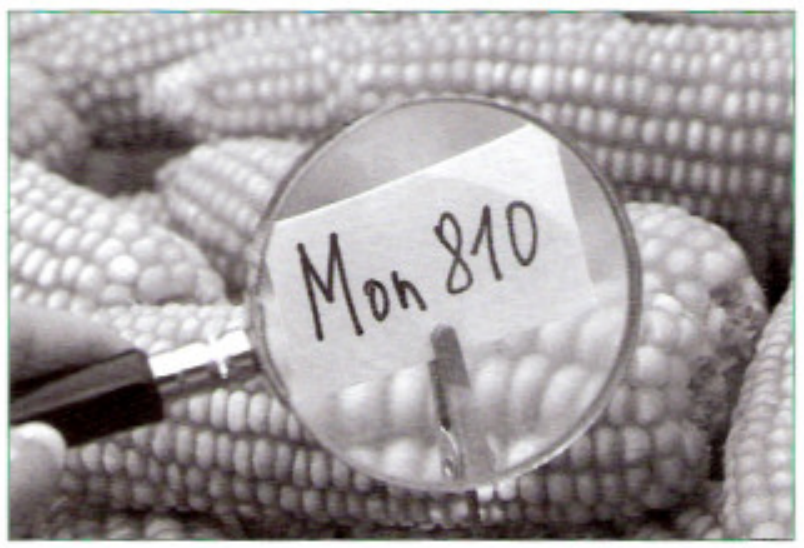

- Florence Durand/SIPA

22

Probabilité et incertitude Mme Dominique DePRINS

\section{2}

Le concept de risque et son évolution Gilles Motet

II - L'incertitude : un facteur omniprésent

38

Quid de la gestion des risques après la constitutionnalisation du principe de précaution? Olivier GODARD

\section{5}

L'investissement de défense face à l'incertitude Carl TrémOUREUX 


\section{\& ENVIRONNEMENT}

\section{Janvier 2010 Numéro 57}

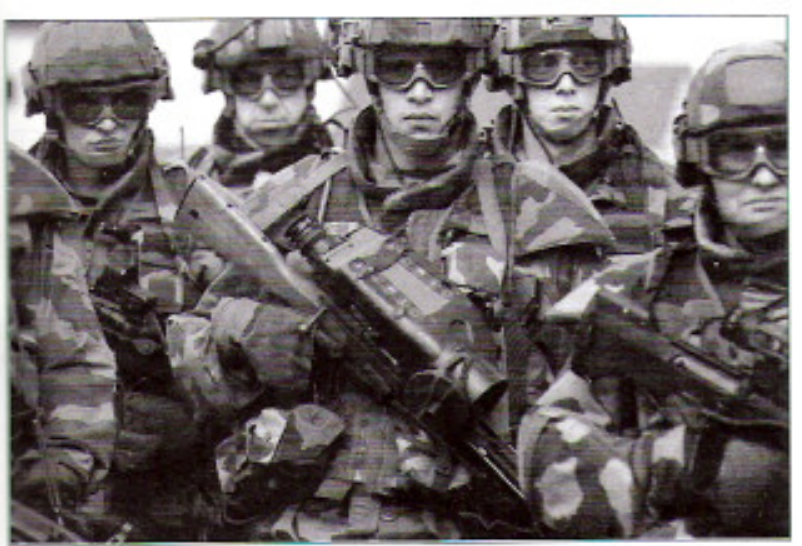

* Zeppelin/SIPA

\section{2}

Les risques chimiques et leur gestion Armand LATTES

\section{9}

CEuvrer dans l'incertitude

Pierre-Michel MENGER

III - Quelle démarche adopter, face à l'incertitude ?

\section{0}

L'incertitude en matière de technologie Sven Ove Hansson

\section{5}

De l'incertitude-obstacle à l'incertitude productive, ou comment traiter les risques potentiels des nano-objets?

Brice LAURENT

\section{2}

De l'incertitude à la précaution : le rôle de la métrologie

Jean-Luc LAURENT et Benoit GAUMONT

\section{2}

Risque et prospective

Thierry GAUDIN

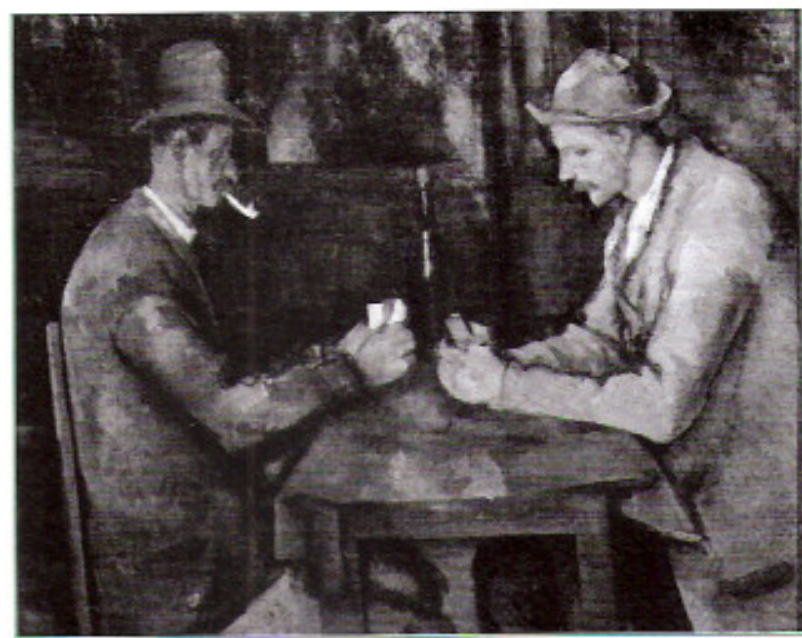

- Photo Josse/LEEMAGE
Ce numéro a été coordonné par

Marie-Josèphe CARRIEU-COSTA 


\section{Titre: Quelques éléments de réflexion sur l'incertitude à travers l'histoire des sciences et des idées.}

(Article paru dans Responsabilité et Environnement, une série des Annales des Mines, n57, janvier 2010, numéro «Faire face à l'incertitude »)

Résumé: L'incertitude a de tous temps accompagné les progrès de la science et de l'industrie: la peur de l'incertain est la face cachée de toute création ou innovation. Une attitude alimente l'autre : anticiper, prévoir, sont producteurs d'innovation, d'imaginaires créateurs, mais aussi de replis, de sécurisations, de "précaution » risquant en retour de tétaniser la société. On retrouve en permanence cette ambivalente vis-à-vis de la science, qui se nourrit de la science elle-même : elle prend ses racines dans certains courants philosophiques des Lumières (Rousseau) ou de l'utopie (Fourier) ; elle est aujourd'hui théorisée par des réflexions philosophiques plus radicales, comme l'hypothèse Gaïa de James Lovelock ou la Deep Ecology du norvégien Arne Naess.

Par Alexandre Moatti $(*)$

$\left({ }^{*}\right)$ Alexandre Moatti, 50 ans, X-Mines, a effectué sa carrière dans le public et dans le privé. En 2003, alors au cabinet de la ministre de la Recherche, il a créé le portail science.gouv.fr, dont il assure la direction de la publication; il est aussi directeur de la bibliothèque numérique d'histoire des sciences bibnum.education.fr. Il a été en 2005-2006 Secrétaire général du projet «Bibliothèque numérique européenne ». Il est depuis 2007 délégué général de la fondation de culture scientifique C.Génial (www.cgenial.org). Il est par ailleurs auteur de plusieurs ouvrages de vulgarisation et d'histoire des sciences chez Odile Jacob (voir son blog www.maths-et-physique.net), maître de conférences à l'ESSEC et à l'EHESS, et président de la Société des amis de la Bibliothèque et de l'histoire de l'École polytechnique (SABIX).

La peur de l'incertitude dans nos sociétés semble affecter le rapport du citoyen à la science, et geler en partie l'avancement de celle-ci, comme celui de l'innovation ou de l'industrie. Les freins mis à la recherche sur l'embryon (2003), l'introduction du principe de précaution dans la Constitution française (2005) ou l'arrêt des expérimentations OGM (2008) peuvent s'analyser comme des expressions au plus haut niveau politique, en France, de cette peur sociétale face à l'incertitude scientifique.

Mais, contrairement à ce qui nous est parfois présenté pour renforcer le propos, il n'y a pas là nouveauté radicale d'une prise de conscience des dangers présumés d'une science triomphante. On peut en trouver trace depuis au moins les Lumières, dans un certain nombre de courants entrant en opposition avec la science ou l'industrie. Les rapports actuels ambivalents entre science et société sont singulièrement éclairés par 
la mise en perspective qu'on peut faire de ces ressorts historiques - nous allons en donner quelques exemples.

\section{«C'est la faute à Rousseau ! ${ }^{1}$ »}

On connaît l'influence qu'a eue et qu'a encore Jean-Jacques Rousseau (1712 -1778) dans la pensée socialiste ou la pensée écologiste. Le Contrat social a inspiré les révolutionnaires jacobins, l'Émile des générations de théoriciens de la pédagogie, «l'état de Nature » et les Rêveries d'un promeneur solitaire de nombreux courants écologistes et environnementalistes.

On sait moins que Rousseau a été un contempteur de la science, notamment dans son Discours sur la science et sur les arts (1750). Selon lui, la science est fille de l'oisiveté et la petite-fille de la richesse. L'astronomie est née de la superstition, les mathématiques de l'avarice et la physique «d'une vaine curiosité ». Il a une vision élitiste de la pratique de la science : elle doit être réservée à quelques esprits géniaux, très peu nombreux - les autres perdent leur temps à l'étude et en oublient leurs devoirs civiques et politiques :

Nous avons des physiciens, géomètres, chimistes, astronomes, poètes, musiciens, peintres; nous n'avons plus de citoyens.

L'étude des sciences est bien plus propre à amollir et efféminer les courages, qu'à les affermir et les animer.

La science n'est bonne à rien, et ne fait jamais que du mal, car elle est mauvaise par sa nature.

Peuples, sachez donc une fois que la nature a voulu vous préserver de la science, comme une mère arrache une arme dangereuse des mains de son enfant; que tous les secrets qu'elle vous cache sont autant de maux dont elle vous garantit (...)

Ces idées sont à mettre dans le contexte d'époque d'opposition à une science "expérimentale" naissante et génératrice d'incertitude - le savant commence à manipuler des objets et des corps, en physique, en chimie, et ne se contente plus de l'observation (astronomie) ou de la "géométrie" (mathématiques). Par ailleurs, certains historiens des sciences ont souhaité relativiser la portée de ces écrits de Rousseau en rappelant qu'il pratiquait lui-même la science ${ }^{2}$, notamment la botanique, à ses heures perdues : il n'en reste pas moins que son discours d'opposition à la science est fort, émanant de quelqu'un qui, justement, n'est pas totalement étranger à la science.

Ce débat sur la science expérimentale - qu'on peut rapprocher des débats actuels entre science et société - se poursuivra en France sous la Révolution où, comme le rappelle le philosophe François Ewald, deux visions de la science s'affrontent : celle

1. « Je suis tombé par terre, c'est la faute à Voltaire ! Le nez dans le ruisseau, c'est la faute à Rousseau !», ritournelle de Gavroche, dans Les Misérables de Victor Hugo (1862).

2. Voir B. Bensaude-Vincent et B. Bernardi (dir.), Rousseau et les sciences, L'Harmattan, 2003. 
de Rousseau, de Marat et des Jacobins, celle de Condorcet, de Lavoisier et de Prieur de la Côte d'Or ${ }^{3}$. C'est la seconde vision qui l'emporte à ce moment-là - la science comme moteur de l'épanouissement et de la Nation - avec la création des écoles scientifiques, Polytechnique notamment en 1794, et qui ouvre la voie au positivisme triomphant de la seconde moitié du XIXe siècle.

\section{@@@@@@@}

Avançant un peu dans le temps, nous choisirons notre deuxième exemple dans les utopies socialistes des années 1810-1830, tout particulièrement celle de Charles Fourier (1772-1837). On connaît là aussi l'influence de ce philosophe sur la pensée socialiste : il est un des premiers à parler de l'amélioration du sort des ouvriers - et c'est un des principaux inspirateurs de Karl Marx. Sa fantasmagorie n'est pas non plus étrangère au mouvement surréaliste d'André Breton et au mouvement anarchiste de Mai $68^{4}$.

La pensée de Fourier est fort complexe, et pour ainsi dire totalement délirante dans certains de ses écrits (voir par exemple Harmonie aromale des astres). À l'inverse de Rousseau, il ne pratique pas la science, même en amateur - mais il éprouve une espèce de fascination médiévale et scolastique pour la théorie de l'attraction universelle de Newton, sans la comprendre d'ailleurs. Le fouriérisme, comme le saint-simonisme, tous deux qualifiés d'« utopies socialistes », puisent aux mêmes sources d'un « newtonisme social ${ }^{5}$ », faisant vœu de transposer l'harmonie du monde céleste à une harmonie du monde humain.

En parallèle à cette fascination de Fourier pour la science, on trouve dans son Détérioration matérielle de la planète une opposition virulente à l'industrie capitaliste, en même temps que les premières idées « écologistes », véritables prémisses d'une « écosophie ${ }^{6} »$ :

Sortir promptement de l'état Civilisé, Barbare, Sauvage, et remédier aux souffrances de la planète.

Préserver de l'excès de population qui est l'un des écueils du monde Civilisé.

Le tableau des ravages de l'industrie civilisée, des fléaux dont elle affecte la superficie de la planète.

Je viens de signaler en industrie une duplicité non moins choquante, celle des nations civilisées et barbares, en collusion pour dégrader la superficie de la planète.

\footnotetext{
3. Claude-Antoine Prieur-Duvernois (1763-1832), officier du génie, homme politique révolutionnaire (membre du comité de Salut public). Un important fonds d'archives UAP-Prieur de la Côte d'Or est déposé à la bibliothèque de l'École polytechnique (voir Bulletin de la SABIX, n8, décembre 1991).

4. On connaît le mot prêté au normalien Georges Pompidou à propos du mouvement de Mai 68 : « Au fond, tout çà, c'était Breton » (rapporté par Julien Gracq, cité par L. Janover, Cent ans de servitude ; Aragon et les siens, Sulliver, 1998)

5. Dans un autre champ scientifique, on a beaucoup parlé en 2009, année Darwin, des interprétations extensives dites du «darwinisme social », pouvant aller jusqu'à appliquer la théorie de la sélection naturelle à l'eugénisme.

6. Voir notamment René Schérer, L'écosophie de Charles Fourier, Ed. Anthropos, 2001. On retrouvera ce néologisme plus loin, à propos de l'écosophie contemporaine - ou philosophie de l'écologie.
} 
On notera au passage que Fourier déplore dans les années 1820 le refroidissement de la planète : il évoque le gel à Nice dans l'hiver 1820, l'accroissement des glaces polaires et alpines - à l'époque de la petite ère glaciaire (env. 1600-1840), le réchauffement climatique était ardemment souhaité.

\section{@@@@@@@}

Il est certes évident que l'opposition à la science ou à l'industrie est bien plus ancienne que l'éclosion des mouvements environnementalistes dans les années 1970. Il est néanmoins intéressant de s'en rendre compte à la lecture de ces deux penseurs influents. De manière complémentaire, à la lumière de Rousseau et de Fourier, nous sommes amenés à formuler deux hypothèses.

L'influence de ces penseurs - ou de l'idée qu'on s'en fait de leur pensée - sur l'inconscient collectif, en même temps que sur les mouvements politiques écologiste ou socialiste, est tellement importante et universelle que leur pensée a été harmonisée voire réduite à un socle à peu près acceptable par chacun de nous, laissant de côté une partie moins connue de leur œuvre, une face cachée. Nous pensons néanmoins première hypothèse - que c'est l'ensemble de l'œuvre de ces deux philosophes, y compris les aspects anti-scientifique ou anti-industriel, qui imprègne, en filigrane, voire de manière résurgente de nos jours, les mouvements politiques d'inspiration écologiste ou socialiste. Et ceci nous fait réfléchir dans la compréhension que nous pouvons avoir de ces mouvements aujourd'hui.

Par ailleurs, ces deux philosophes s'inscrivent chacun dans un mouvement de pensée plus vaste - Rousseau dans les Lumières et Fourier dans l'utopie socialiste. On a vu que l'idéologie rousseauiste s'opposait, dans le rapport à la science, à celle d'un autre philosophe des Lumières, par ailleurs mathématicien réputé, Condorcet. De la même manière, Fourier s'oppose aux idées du comte de Saint-Simon (1760-1825) : nés dans le même creuset de l'utopie, maniant des idées analogues, les deux mouvements s'entrechoquent entre 1820 et 1870 et se disputent disciples - militants dirait-on de nos jours - issus eux aussi du même creuset, notamment l'École polytechnique ${ }^{7}$. Et, comme les tenants de Condorcet, malgré les idées de son collègue Rousseau, ont mené la révolution scientifique française de 1795 à 1820, le saint-simonisme conduira à - et conduira - la seconde révolution industrielle ${ }^{8}$, celle du chemin de fer dans les années 1850. Le saint-simonisme catalyse de manière positive une certaine vision de la science et de l'industrie : mais justement le fouriérisme, inspirateur du marxisme,

\footnotetext{
7. Le mouvement saint-simonien sera porté, après la mort de Saint-Simon, par de célèbres polytechniciens : Prosper Enfantin (le "père Enfantin", 1796-1864), Michel Chevalier (1806-1879), ingénieur des mines, conseiller influent de Napoléon III ou Paulin Talabot (1799-1885), fondateur du réseau ferroviaire P.L.M., I'un des premiers chefs d'entreprise industrielle - l'un des premiers représentants du "patronat". Mais le fouriérisme n'est pas en reste et va chercher ses troupes dans le même vivier : le polytechnicien Victor Considerant (18081893) portera sa vie durant la doctrine du maître, comme l'ingénieur des mines Abel Transon (1805-1876), mathématicien, transfuge du saint-simonisme.

8. On ne peut pas manquer de faire un parallèle peut-être abusif mais particulièrement tentant (d'autant qu'il a déjà été fait) de relecture de ces deux mouvements socialistes du XIX ${ }^{\mathrm{e}}$ siècle, en comparant le saint-simonisme à la « nouvelle gauche » et le fouriérisme à la gauche traditionnelle ou radicale.
} 
n'est-il pas l'éternelle face cachée d'un saint-simonisme triomphant, inspirateur d'une France d'ingénieurs ? Cette intrication nous amène à notre deuxième hypothèse : toute doctrine nouvelle qui devient dominante - celle des Lumières et de l'épanouissement par la connaissance philosophique et scientifique ou celle de l'industrie saint-simonienne triomphante - produit en son sein sa propre opposition, sa propre face cachée. Ainsi, la Nation secrète en ses propres élites une opposition à la tendance dominante : de nos jours, à l'ère d'une « technoscience » qui s'est infiltrée dans tous les domaines de la vie quotidienne, de nombreux chercheurs, ou de nombreux ingénieurs, formés à la science, appartiennent à un écologisme politique et militant ; c'est aussi parce que ces idées sont portées au niveau des élites qu'elles ont une telle résonance dans l'opinion.

\section{@@@@@@@}

Mais poursuivons notre voyage dans le temps, et après Rousseau et Fourier intéressons-nous à un personnage beaucoup plus obscur, Eugène Huzar (1820-1890). Jean-Baptiste Fressoz a récemment exhumé ses œuvres, notamment La fin $d u$ monde par la science $(1855)^{9}$. En ce début de la seconde révolution industrielle, Huzar, homme éclectique et fortuné, lance un avertissement sur l'« orgueil de la science » et stigmatise - à nouveau - les dangers de l'industrie et de la "science expérimentale », celle qui en vient à utiliser et modifier la Nature et pas seulement l'expliquer - notre " technoscience » en quelque sorte. Il utilise des controverses de son époque (inondations dues aux déforestations, accidents meurtriers de chemin de fer, craintes liées aux débuts de la vaccination,...) pour dénoncer une société productrice de risque et d'incertitude. Loin d'être un ouvrage de science-fiction, ce pourrait être un bréviaire avant l'heure de la société du risque, ou plutôt de la société du risque tel qu'accepté avec la révolution industrielle du XIXe siècle. Il nous montre que les débats autour de la «technoscience » ne sont pas aussi radicalement neufs qu'on voudrait nous le faire croire, et ainsi infirme le fait que seuls certains visionnaires ou hommes de communication éclairés de la fin du $X X^{\mathrm{e}}$ siècle nous auraient ouvert les yeux sur le risque industriel. Certes, la dramatisation actuelle aide à une prise de conscience plus générale, et les risques sont sans conteste plus grands à notre époque, mais l'histoire de l'environnement n'est pas celle d'une prise de conscience

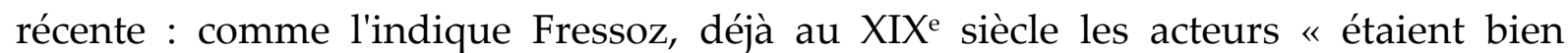
conscients de l'incertitude gigantesque produite par leurs choix technologiques, ils étaient bien conscients de défaire leurs environnements et ils ont sciemment décidé de passer outre (...) Il apparaît trompeur de raconter la révolution industrielle comme l'histoire de sociétés modifiant de manière inconsciente leurs environnements et réalisant a posteriori leurs erreurs ». On notera aussi chez Huzar l'idée d'une « édilité planétaire », gouvernement mondial de savants, une sorte de GIEC ${ }^{10}$ Prix

9. Eugène Huzar, La fin du monde par la science (1855), Introduction J.B. Fressoz, postface B. Latour, Éditions Ère, 2008.

10. Groupe d'experts intergouvernemental sur l'évolution du climat, créé en 1988 par l'ONU, prix Nobel de la paix en 2007. 
Nobel avant l'heure : ce n'est pas une anticipation, mais plutôt une indication sur la similarité des idées qui pouvaient germer à l'époque.

\section{Écologie profonde}

$\mathrm{Au} X X^{\mathrm{e}}$ s., une véritable philosophie de l'écologie - parfois appelée écosophie - fait son apparition, d'abord dans le monde anglo-saxon, et s'appuyant elle aussi sur la science. Nous présentons sommairement ci-après deux de ces théories, parmi d'autres, l'hypothèse Gaïa et la Deep Ecology.

James Lovelock (né en 1919) est un scientifique britannique, " géophysiologiste », comme il se désigne lui-même - c'est-à-dire physiologiste de la planète Terre. Il lance en 1972 "l'hypothèse Gaïa », du nom donné par les Grecs à la déesse Terre. Cette théorie décrit notre planète comme vivante, à l'instar de ses occupants : à la différence des autres planètes, elle régule sa température par le truchement de l'effet de serre $^{11}$, elle fait partie d'une biosphère où elle est en interaction avec les autres éléments vivants, du micro-organisme végétal au plus gros mammifère, en passant par l'être humain. Le biomorphisme est poussé plus loin par Lovelock quand il imagine que « la Terre devient moins résistante en vieillissant » ou que le réchauffement climatique s'assimile à une " fièvre » de notre planète. Il estime qu'il est trop tard pour le développement durable, et qu'il convient d'opter pour un « repli durable ». D'un point de vue épistémologique, il s'oppose à une pensée atomiste et « réductionniste », à la Descartes, qui nous aurait asservis au dogme de la distinction sujet-objet. Il prône une philosophie holistique ${ }^{12}$, qui considère les éléments vivants dans leur tout et dans leurs interrelations, une approche «top-down ». Il va jusqu'à étayer son écosophie en utilisant le principe des états intriqués de la physique quantique $^{13}$ : tous les éléments vivants, y compris la planète elle-même, interagissent entre eux, même à distance.

L'approche de Lovelock, qui depuis 2000 suscite un intérêt croissant de la part de certains scientifiques - et bien sûr de certains mouvements écologistes - a ceci d'intéressant qu'elle se distingue des courants écologistes dominants - il revendique

\footnotetext{
11. Rappelons que, contrairement à une idée fortement ancrée, l'effet de serre est indispensable à la vie sur Terre, puisqu'il permet de faire passer la température de surface de la planète de $-18^{\circ} \mathrm{C}$ (température de rayonnement du corps noir constitué par la Terre, à distance donnée du Soleil) à $+15^{\circ} \mathrm{C}$ (grâce à la réflexion par l'atmosphère en retour vers la Terre d'une partie de ce rayonnement). C'est, bien sûr, l'augmentation de l'effet de serre qui pose problème.

12. Le "holisme" (de holos, gr.: le tout) est une doctrine philosophique non spécialement rattachée à l'écologie, mais utilisée par cette dernière. Suivant cette doctrine récente (années 1920), et de manière simplifiée car elle a plusieurs acceptions, l'étude du tout prime sur l'étude de ses constituants : un système ne peut être étudié que dans le cadre global dans lequel il s'inscrit. En sociologie, le holisme s'oppose à l'individualisme de nos sociétés.

13. La physique quantique, depuis les interprétations extensives du principe d'indétermination d'Heisenberg jusqu'aux broderies sur le chat de Schrödinger - comme la théorie des mondes multiples - a depuis les années 1920 été source d'inspiration pour nombre de théories connexes. Depuis les années 1980, c'est l'un de ses principes fondamentaux, l'intrication des particules, qui est très prisé dans certaines approches holistiques ou écologistes. Leur apparition a été concomitante : c'est entre 1965 et 1985 (des inégalités de Bell aux expériences d'Aspect) que le principe d'intrication a été confirmé comme intrinsèque à la description quantique de la réalité.
} 
cette petite musique différente en en attribuant l'origine à sa solide formation scientifique : par exemple, il est pro-nucléaire et accorde une confiance très limitée aux énergies renouvelables; de même, il estime que le réchauffement climatique d'origine solaire est beaucoup plus grave pour l'avenir de Gaïa que le réchauffement d'origine anthropique.

\section{@@@@@@@}

Arne Naess (1912-2007) est quant à lui un philosophe norvégien, théoricien de la Deep Ecology, ou écologie profonde. Cette doctrine prend appui sur la science et a elle aussi une interprétation fort extensive de la physique quantique - cette " nouvelle science » venant fort opportunément à l'appui de la nouvelle vision du monde par l'écologie. Ainsi, la physique aurait abandonné une vision matérielle de particules bien définies dans l'espace, au profit d'un flux permanent d'échanges d'énergie. Comme chez Lovelock, la vision cartésienne distinguant clairement sujet et objet serait dépassée, laissant la place à une vision holiste: l'homme n'est pas une entité isolée, mais un des constituants du monde qui l'entoure, et en échange permanent avec son environnement, formant la «toile de la vie » (Web of life). La biologie serait la science-reine, ayant détrôné la physique dans la primauté de description de la nature et du monde ${ }^{14}$.

L'impossibilité de distinction entre observateur (sujet vivant) et entité observée (objet inanimé) correspond à une interprétation fort extensive au niveau macroscopique du principe d'Heisenberg de la physique quantique, souvent citée dans les doctrines se rattachant à l'écologie profonde. Cette interprétation est parfois poussée à l'extrême avec de curieuses conclusions. Par exemple, certains écologistes ${ }^{15}$ dénient à l'U.S. National Forest Service le bien-fondé de modélisation des écosystèmes forestiers : la compréhension de la nature passe nécessairement par l'immersion dans la nature. " La carte n'est pas le territoire »: la Terre est un organisme vivant et doit être étudiée en conséquence - toute modélisation, par définition externe à la nature, est invalide. On retrouve là une des réticences majeures de ces mouvements à l'égard de la technologie informatique, support d'une «société cybernétique » qui isole l'homme dans la virtualité et le coupe de son environnement réel. Dans le même ordre d'idées, l'écologie profonde veut «questionner la technologie », et rappelle en la développant l'identité sémantique entre technologie et technocratie, l'une étant décrite comme le ressort du pouvoir exercé par l'autre.

\section{@@@@@@@}

Nous terminerons notre lecture engagée de l'utilisation de la science dans certains courants de pensée à travers l'histoire par un roman de science-fiction - on sait bien

\footnotetext{
14. Il n'est pas banal de constater, si l'on suit cette idée de l'écologie profonde, qu'une « philosophie de la nature » a chassé l'autre : la philosophie de la Nature au XXI ${ }^{\mathrm{e}}$ siècle, c'est celle qui s'intéresse à la nature au sens environnemental, à savoir l'écologie ou la biologie; la philosophie de la nature au XVII ${ }^{\mathrm{e}}$ et au XVIII siècles - la Naturphilosophie - était le terme utilisé pour désigner la physique (terme qui n'était pas encore utilisé).

15. Voir notamment William Devall \& George Sessions, Deep ecology (1985). Gibbs Smith, Salt Lake City.
} 
que ce qui la distingue, c'est qu'elle ne se produit jamais, ou alors dans une autre planète! Dans Le Meilleur des mondes d'Aldous Huxley (1932, Brave New World en anglais $\left.{ }^{16}\right)$, l'Administrateur mondial Mustapha Menier, auparavant physicien - c'est un point signifiant -, explique, en épilogue tenant lieu de clef au roman, comment il tire les ficelles de cette société du bonheur totalement aseptisée :

Nous ne voulons pas changer. Tout changement est une menace pour la stabilité. C'est là une autre raison pour que nous soyons si peu enclins à utiliser des inventions nouvelles. Toute découverte de la science pure est subversive en puissance; toute science doit parfois être traitée comme un ennemi possible. Oui, même la science [...]

La science est dangereuse; nous sommes obligés de la tenir bien soigneusement enchaînée et muselée. [...] Voilà pourquoi nous limitons avec tant de soin le rayon de nos recherches [...] Cela n'a pas été une fort bonne chose pour la vérité bien entendu. Mais ç'a été excellent pour le bonheur.

Le personnage d'Huxley nous explique que le monde est passé d'une civilisation auparavant fondée sur la vérité de la science et la beauté de l'art à une civilisation fondée sur le confort et le bonheur. Dans cette dystopie, ou utopie "négative ", immense succès de son auteur, la civilisation s'est fermée les voies d'une science libre car celle-ci est jugée trop génératrice d'incertitude. Prenons garde à ne pas nous bâtir un «meilleur des mondes ».

16. La traduction française de ce titre anglais (à mon sens pourtant plus parlant) n'est pas neutre : le titre anglais est une référence littéraire à Shakespeare (premier vers de La Tempête), comme le titre français est une référence à la phrase « Tout est pour le mieux dans le meilleur des mondes possible » (Voltaire, Candide), phrase d'ailleurs mise en exergue par Huxley sur la page de garde. Ceci nous ramène aux Lumières et aux utopies, avec lesquelles nous avons commencé notre article. 